\title{
LA DIVISION TERRITORIAL EN COMARCAS DEL PAIS VALENCIANO
}

$311: 352.071(-201)$ (46. Valencia)

por

Ignacio Ballester Ros

SUMARIO: I. INTRODUCCION.-II. LA COMARCA: SU CONCEPTO Y CRITERIOS DE ORDENACION.-III. PRINCIPALES DIVISIONES COMARCALES DEL PAIS VALENCIANO.-IV. LA DIVISION COMARCAL DE RIBERA Y CABRER.

\section{INTRODUCCION}

Recientemente se han publicado en Valencia dos monografías sobre un mismo tema: La comarcalización del País Valenciano.

La primera de ellas, editada por el Servicio de Estudios y Planificación Social de la Diputación Provincial de Valencia, con la colaboración de la Delegación Interprovincial, en el antiguo Reino de Valencia, del Instituto de Estudios de Administración Local, se titula Taula Redona sobre la comarcalització al País Valenciá y contiene los trabajos presentados a la Mesa Redonda celebrada sobre este tema en los días 5 a 9 de noviembre de 1979.

Las comunicaciones presentadas a dicha Mesa Redonda fueron 19 , todas ellas referidas a distintos aspectos de la comarcalización del País Valenciano. Con expresión de sus autores y temas se enumeran seguidamente: 
Ferrán Vidal i Gil: La comarcalització del País Valenciá en el procés d'institucionalització autonómica.

Vicent Ventura: Raons d'una comarcalització y condicions per a una altra.

Max Cahner: Condicionaments politics de la divisió territorial.

Vicenç M. Rossell'i Verger: Les Comarques valencianes: Un enfocament geográfic.

Pedro Pérez Puchal: La comarcalització del territori valenciá.

Bernardí Cabrer: Téchniques de comarcalització.

Antoni Rico Gil, Joaquín Mafé Sanantonio, Francesc Mas Verdú y Manuel López Estornell: La delimitació comarcal des d'una perspectiva económica.

Prevasa: Propuesta de comarcalización del País Valenciano desde el punto de vista de la acción regional.

Joan F. Mateu Bellés: Subdesenvolupament $i$ comarcalització: El cas del nord valenciá muntanyós.

Josep M. Bernabé Mestre: Els centres comarcals al sud del País Valenciá.

Josep M. ${ }^{2}$ Tortosa: Criteris de comarcalització (Entre el pragmatisme y la crítica).

Joan Francesc Mira: El meu poble $i$ jo (Notes per mirar la Comarca amb uns altres ulls).

Josep-Vicent Marqués: De la impossibilitat actual de la Comarca "espontánia» a l'assumpció de la Comarca com a decisió politica.

Antoni Ferrer: Una experiència de treball urbanistic a nivell comarcal a València.

Albert Peñín: Ambits urbanistics supramunicipals.

Joan Ferrán Martínez y Navarro: Criteris per a l'ordenació sanitaria territorial del País Valenciá.

Col. Lectiu de Mestres La Safor: Un intent d'organització pedagógica comarcal.

Lluís Aguiló Lúcia: Comarques: Governacions i Diputacions.

Vicent Franch i Ferrer: Les Comarques i la futura llei electoral.

La segunda, titulada Los desequilibrios espaciales: Una comar. calización del País Valenciano, de la que son autores María José Ribera Ortún y Bernardí Cabrer Borrás, ha sido editada por la Delegación Interprovincial, en el antiguo Reino de Valencia, del Instituto de Estudios de Administración Local.

Desde el siglo xvin han sido numerosos los estudios realizados sobre comarcalización de la Región Valenciana. En los últimos de- 
cenios, sin embargo, y, de manera especial, en el último quinquenio, aunque más singularmente desde el establecimiento del Ente Preautonómico del País Valenciano y la promulgación de la Constitución española de 6 de diciembre de 1978, se ha despertado un extraordinario interés por este tema entre especialistas y estudiosos valencianos-geógrafos, historiadores, demógrafos, estadísticos, matemáticos, sociólogos y economistas-y también políticos.

Fruto de este renacer del interés por este tema son, entre otras, las dos obras reseñadas, que vamos a sintetizar, tanto desde el punto de vista conceptual, como con referencia a los criterios aplicados a la configuración de las Comarcas y a las principales divisiones comarcales formuladas.

La preocupación que se siente en el País Valenciano por la estructura territorial de la futura Comunidad Autónoma justifica sobradamente este comentario.

\section{LA COMARCA: SU CONCEPTO Y CRITERIOS DE ORDENACION}

La Comarca es un hecho. Está presente en nuestra vida. La conciencia del pueblo la percibe. Cada habitante se define como residente en un Municipio y oriundo de una Comarca.

La Comarca, en una primera etapa, ha sido configurada con un criterio básicamente geográfico, como la genuina expresión de la unidad natural, que el relieve, la hidrografía, juntamente con los demás rasgos fisiográficos y geográficos definen.

En una etapa subsiguiente, que corresponde a una estructura política fuertemente centralizada, se la concibe como un Ente territorial, en el que se ubican una serie de servicios públicos comunitarios, comunes a una pluralidad de Municipios, que configuran aquélla; y así se habla del Municipio-Comarca como el ideal aplicable al sostenimiento de los servicios que no pueden ser mantenidos en el área de cada término municipal.

En la actualidad, la Comarca es considerada, además, como un Ente político-administrativo, con funciones de representación y participación, al propio tiempo que como expresión de una homogeneidad socioeconómica en la que una población se comporta con arreglo a sus formas de vida propias.

La Constitución española establece en su artículo 137 que el Estado se organiza en Municipios, Provincias y Comunidades Autó- 
nomas. Y en el artículo 141-3 se prevé la posibilidad de que puedan crearse Agrupaciones de Municipios, diferentes de las Provincias.

Estas Agrupaciones de Municipios pueden ser las Comarcas.

Esta definición de Comarca como Agrupación de Municipios contrasta con la concepción centralista de la Comarca, antes apuntada, como ordenación del territorio para la realización de determinadas actuaciones técnicas.

Según la concepción de la Constitución, el territorio español tendrá una división de primer grado, que son las Comunidades Autónomas; otra, de segundo grado, las Provincias; otra, de tercer grado, las Comarcas; y, finalmente, otras de cuarto grado, los $\mathrm{Mu}$ nicipios.

Toda división territorial ha de fundamentarse sobre bases sólidas y objetivas para que sea auténtica y perdurable. Se trata de una cuestión compleja, ya que en ella influyen factores de muy diversa índole-geográficos, históricos, lingüísticos, sociales y económicos-; pero, en el fondo, la decisión ha de ser política.

Cuando se inician los estudios de comarcalización de un territorio es necesario primeramente concretar los objetivos que se persiguen, así como la utilidad que va a derivarse de ella, en particular, desde el punto de vista socioeconómico, ya que, en definitiva, lo que se pretende es la potenciación social y económica del territorio mediante un mejor aprovechamiènto de los recursos.

Hay quien piensa que la afirmación de la Comarca como Ente político autónomo sería en perjuicio del Municipio; y que si se prescindiera de las Provincias y de las Diputaciones provinciales, la Comarca adquiriría una personalidad que sería básica para la organización territorial del País Valenciano.

Es conveniente disponer de mecanismos de ordenación e institucionalización del territorio en Comarcas y fijar las formas de mantenimiento de las relaciones de las Comarcas con otras instituciones, a nivel de Estado, a nivel de Comunidad Autónoma, a nivel de Provincia.

En la Mesa Redonda, cuyas comunicaciones contiene la primera de las monografías reseñadas, se ha estudiado la Comarca desde los siguientes aspectos:

a) Antecedentes y planteamiento de la cuestión.

b) Métodos y técnicas utilizadas para la ordenación comarcal del territorio.

c) Enfoques sectoriales. 
d) Implicaciones jurídico-políticas e institucionales de la comarcalización.

Han sido muy variados los criterios de comarcalización del territorio que han sido utilizados en el pasado:

1. El primero de ellos, el criterio «geométrico» o de «uniformidad superficial», es el menos digno de tenerse en cuenta, ya que no está claro que puedan tener la misma extensión las Comarcas superpobladas del litoral que las despobladas del interior. De otra parte, es conveniente que no se configuren Comarcas grandes, que alejen a los administrados del centro ordenador del espacio territorial.

2. El criterio "demográfico» es el más racional, siempre que se definan previamente las áreas metropolitanas, que lógicamente han de contener una mayor población que las Comarcas rurales.

3. El criterio "histórico» es eficaz cuando existe una tradición de conciencia comarcal, y puede servir de guía adecuada para tener en cuenta la idiosincrasia y hábitos de vida de los habitantes.

4. El criterio "geográfico" o "fisiográfico» es particularmente importante, porque el relieve y las cuencas hidrográficas configuran verdaderas unidades naturales que influyen en el asentamiento de las poblaciones, en la utilización de los recursos naturales, en el desarrollo económico, en la disponibilidad de comunicaciones y accesibilidad a los núcleos poblados, en los mercados y en las formas de vida.

5. El criterio «idiomático» resulta poco aprovechable.

6. El criterio "económico» exige la disponibilidad de buenas estadísticas referidas a áreas muy reducidas, básicamente los $\mathrm{Mu}$ nicipios, lo que no siempre es posible. A partir de las producciones, las distancias, los mercados, los impuestos, el gasto público y otros factores se pueden construir modelos matemáticos, que no siempre dan resultados plenamente satisfactorios.

7. El criterio «socioeconómico», dentro de cuyo concepto entran las relaciones de vecindad, la densidad de población, las formas de vida y las afinidades culturales y lingüísticas, además de la estructura económica.

8. El criterio "nodal", de cohesión en torno a los núcleos urbanos, muy real y efectivo en la actualidad, no conviene llevarlo hasta sus últimas consecuencias, ya que supondría la desaparición de las Comarcas. 
Como se observa, existen distintas concepciones básicas para el establecimiento de divisiones comarcales adecuadas. Sin embargo, la cuestión no ha tenido una solución satisfactoria. La tradición popular responde a realidades obvias; pero no ayuda a delimitar las Comarcas como es necesario ni se ajusta siempre a las perspectivas de la vida actual.

Los criterios apuntados en los párrafos anteriores coinciden en el fondo; pero discrepan en el detalle y en la eficacia. Por ello, resulta necesario un profundo estudio por parte de los especialistas para llegar a unas conclusiones que hagan viable la división comarcal.

Las divisiones comarcales al uso en el País Valenciano adolecen de una falta de discusión y de explicación de objetivos y criterios políticos en que se basan. Dos son los objetivos que deben considerarse necesarios:

a) La división comarcal no es un fin en sí misma, sino que debe ir ligada a una reforma político-administrativa.

b) El criterio de participación ha de ir ligado al de eficiencia, porque de lo contrario queda vacío de contenido el primero.

Las Entidades locales tienen un doble carácter, como entidades representativas y de participación y como organizaciones prestadoras de servicios públicos.

Se critican las actuales divisiones comarcales por el tamaño que tienen las Comarcas, tanto física como poblacional y por su acentuado uniformismo, ya que dan el mismo tratamiento al espacio metropolitano que a la Comarca rural.

Conviene tener muy en cuenta, al estudiar la configuración comarcal del País Valenciano, que en el decenio 1960-1970 el País Valenciano acentuó su proceso de industrialización y de urbanización, así como el fenómeno de la segunda residencia, que se desarrolló muy notoriamente el turismo, tanto receptivo como interior, que hubo profundos movimientos migratorios del campo a la ciudad y que igualmente se han desarrollado los medios de transporte y la tecnología.

En la comarcalización del País Valenciano habrá que partir, en principio, de la unidad "término municipal», aunque comprende muchas veces un espacio poco coherente desde el ángulo de la geografía, el idioma, las relaciones de mercado y las comunicaciones.

De otra parte, el propósito de confirmar a la Comarca como una entidad viable, a escala civil, y de coordinar el conjunto de 
Comarcas con una expectativa homogénea y dinámica, aconseja buscar el equilibrio entre la extensión superficial y el número de habitantes.

Hay que descartar también las «microcomarcas» por más personalidad geográfica, histórica o filológica que tengan; e igualmente será muy conveniente desdoblar las «macrocomarcas» tradicionales, de tamaño excesivo.

También conviene cuidar mucho la nomenclatura de las Comarcas, teniendo presente que la Comarca no es un nombre sino la tierra y los hombres que en ella viven.

Hemos de resaltar el hecho de que a lo largo de la historia de la Región valenciana no ha existido una institución política comarcal, que, de otra forma, habría contribuido mucho a establecer las bases de una adecuada división comarcal del territorio.

En el momento actual se trata de contribuir a la institucionalización de la Comarca como hecho administrativo y a determinar tanto su espacio físico como su contenido político, administrativo.

Nuestra división comarcal ha de concordar con la experiencia del pueblo y con las hipótesis contrastadas de los estudiosos. No es fácil encontrar una división administrativa comarcal que responda a la realidad geográfica y humana. No se trata de volver a las circunscripciones históricas, fruto a menudo de un complicado conjunto de intereses condicionados por la expansión de los dominios señoriales durante la Edad Media o en los siglos posteriores, ni tampoco de hacerlas coincidir con puras Regiones geográficas, ni. con las Comarcas naturales condicionadas por la fisiografía, la hidrografía, el clima y la biogeografía, más que por las realidades económicas y sociales de carácter territorial. Es lógico que la división comarcal tienda a estructurar un Ente administrativo más que a facilitar la descripción geográfica del territorio.

Se considera que, en definitiva, una división territorial comarcal debe establecerse sobre la base de la coordinación de:

a) Criterios uniformes respecto al conjunto del Estado, que hagan, por ejemplo, incuestionables las Provincias y las Diputaciones.

b) La concepción centralizada o descentralizada del futuro Gobierno de la Comunidad Autónoma del País Valenciano.

c) El planteamiento de prioridades en orden a una política de recuperación nacional, que tenga en cuenta las dos zonas lingüís. ticas de la Región. 
La primera opción puede ser una imposición centralista del Estado, que no permita otra cosa que la subdivisión de las Provincias en Comarcas vacías de todo contenido político.

La segunda opción no ha de impedir que la división territorial se haga de forma que ninguna Comarca, Ciudad, Villa o Pueblo puedan considerarse marginados. Cuando se piensa en un País Valenciano descentralizado, nos viene a la mente la "Región», o si queremos recuperar una palabra histórica, las "Gobernaciones», o sea, la agrupación de Comarcas que constituyen un Ente político. No cabe pensar que tres Diputaciones provinciales puedan ser sustiuidas por 32 Consejos Comarcales. La descentralización administrativa pudiera estar en las Gobernaciones y en las Comarcas como una concepción dual de la división administrativa. Es cierto que la Comarca, centrada casi siempre en un mercado agrícola, no ha tenido una plasmación administrativa; pero los profundos cambios registrados en la estructura económica han modificado el papel de los mercados, y la intensa industrialización de muchas zonas, el despoblamiento en otras y la evolución de los sistemas y vías de comunicación han hecho perder a la Comarca su carácter de unidad económica.

Los problemas locales habrian de tener una consideración político-administrativa a través de las Comarcas que, al propio tiempo, podrían servir de freno a cualquier tipo de centralismo, estatal o autonómico.

La división comarcal, en suma, habrá de hacerse desde arriba, desde la Generalidad del País Valenciano, con todos los asesoramientos necesarios, a fin de llegar a una solución útil.

\section{PRINCIPALES DIVISIONES COMARCALES DEL PAIS VALENCIANO}

Hasta 16 divisiones comarcales del País Valenciano se han propuesto atendiendo a los diversos criterios aplicables.

Interesa subrayar que, por encima de cualquier sospecha de veleidades diferencialistas, nuestra división comarcal concuerda con la experiencia del pueblo y con las hipótesis de los estudiosos, tanto en los clásicos-Viciana, Escolano, Cavanilles-como en los modernos-Llorente, Mateu y Llopis, Beut Belenguer, Sanchis Guarner, Querol Roso, Cano Maroués, López Gómez, Roselló y otros-. 
La personalidad histórica ha estado supervalorada en ciertas divisiones comarcales, como la de Cavanilles-1787-, el gran precursor que se apoyó en la fisiografía y en la oposición entre los señoríos y las tierras de realengo. Lo propio hace en 1932 MateU y LLOPIS y más recientemente SANCH IS GUARNER.

Para el País Valenciano, con una superficie de $23.000 \mathrm{~km}^{2}$, podrían sugerir Comarcas de entre 200 a $1.000 \mathrm{~km}^{2}$ de extensión superficial, con un mínimo de 10.000 habitantes cada una. De esta forma se configurarían unas 30 Comarcas.

Joan SOLER Y RIBER trata de identificar áreas comerciales y Comarcas, basándose en las encuestas realizadas en 1950 por la Escuela de Geografía de la Universidad de Zaragoza, criterio que aplica, en 1964, a la Región Valenciana. Este criterio ha perdido valor dada la evolución de los mercados tradicionales en las últimas décadas.

Emilio Beut y Belenguer considera la lengua como el elemento primordial para la diferenciación de Comarcas.

Numerosas son las referencias bibliográficas y las noticias sobre cada una de las divisiones comarcales propuestas, que se hacen en la primera monografía que comentamos; pero queremos prestar una mayor atención a dos divisiones comarcales, las de Pedro Pérez Puchal y la de Prevasa, a las que se dedican amplias comunicaciones.

Pérez Puchal propone una división comarcal del territorio valenciano, con vistas a una demarcación administrativa que, superando la excesivamente grande en Provincias, existente desde 1833, se acomode mejor al futuro gobierno autónomo del País Valenciano. Se las dotará del equipo idóneo, a las que alcancen los servicios públicos y al público desde ciudades cabeceras de Comarca. Para aquéllas que resulten demasiado grandes o demasiado pobladas, se sugiere una división en Subcomarcas o Distritos, que podrían jerarquizar los servicios al tenor del umbral demográfico de cada circunscripción.

Pérez Puchal analiza los diversos criterios aplicables para la configuración de las Comarcas-uniformidad superficial o demográfica, histórico, fisiográfico, económico, nodal o funcional, si bien adopta en definitiva un criterio geográfico, que aglutina y une, en síntesis, a todos los demás. De esta manera configura 25 Comarcas, con una superficie media de $930 \mathrm{~km}^{2}$ y unos 83.000 habitantes de población media.

Estas Comarcas se subdividen en 52 Subcomarcas o Distritos. 
Para cabeceras de Comarca elige Municipios de mayor población, de fácil accesibilidad, que sean buenos nudos de comunicación. En las zonas de despoblación se elige un Municipio de población estable, que tenga industria, aunque sea agrícola, y ocupa una posición central respecto a la demarcación.

Las Comarcas y Subcomarcas que configura son éstas:

PROVINCIA DE CASTELLON

L'ALT MAESTRAT

Els Ports de Morella

Alt Maestrat

L'Alcalatén

BAIX MAESTRAT

Dictricte de Vinarós

Districte de Benicarló

Baix Maestrat Septentrional

El Pla del Arc

La Ribera de Torreblanca

CONCA ALTA DEL MILLARS O ALT MILLARS
LA PLANA DE CASTELLO
La Plana Baixa
La Plana Alta
Espadán

EL ALTO PALANCIA

PROVINCIA DE VALENCIA

EL RINCON DE ADEMUZ

\section{LOS SERRANOS}

EL CAMP DE LLIRIA

Camp de Lliria

La Ribera del Turia

EL BAIX PALANCIA O CAMP DE SAGUNT

L'ALTIPLA CASTELLANO-VALENCIA

Campo de Requena

Campo de Utiel
LA HOYA DE BUNOLL

La Hoya de Buñol

Districte de Cheste a Toris

L'AREA METROPOLITANA DE VA. LENCIA

L'Horta de Valencia

El Pla de Quart

La Calderona

La Albufera

EL CAROCHE O CAROIG

Valle de Ayora

Las Gargantes del Júcar

Enguera-La Canal de Navarrés

LA RIBERA ALTA DEL XUQUER

Els Alcaláns

Districte de Carlet

Districte d'Alberic

Districte d'Alzira

LA RIBERA BAIXA

LA COMARCA SETABENSE

Xátiva-La Costera

La Vall de Montesa

\section{LA SAFOR}

L'Horta de Gandía

La Valldigna

\section{LA VALL D'ALBAIDA}

Districte d'Ontinyent

Districte d'Albaida 
PROVINCIA DE ALICANTE

\section{LA SERRA D'ALCOI}

Foia y Valls d'Alcoi Corredor de Banyeres

La Foia de Castalla

La Canal de Xixona

\section{EL MARQUESAT DE DENIA}

Les Valls de Pego

La Marina Septentrional

\section{LA MARINA}

La Marina

Les Serres de la Marina

EL CAMP D'ALACANT

LA VALL DEL VINALOPO

L'Alt Vinalopó

El Vinalopó Mitjá

EL CAMP D'ELX O BAIX VINALOPO

EL BAJO SEGURA O CAMPO DE ORIHUELA

La investigación efectuada por PrEvasA consiste en la realización de una propuesta de comarcalización del País Valenciano que en todo momento trata de ser coherente con el principio inspirador de la misma, es decir, la acción regional como opción política indispensable para corregir y revisar los graves desequilibrios territoriales que el propio proceso de crecimiento conlleva.

La cuestión básica se centra en la metodología a aplicar, puesto que los criterios de homogeneidad y heterogeneidad son determinantes a la hora de definir áreas espaciales en las que sea viable la acción regional: las Comarcas. Si en los intentos de regionalización la segunda de estas opciones es clara, en la realización de una comarcalización al partir del Municipio como unidad básica, se diluye notablemente la efectividad de dicha metodología. Por ello, el concepto de dependencia funcional, con las interrelaciones que de él se derivan, cobra un especial interés que hace necesaria su presencia en consonancia con los objetivos perseguidos.

La política de acción regional trata de corregir el desigual desarrollo de las Regiones y de las Comarcas. Se basa en los principios de que la planificación regional es una necesidad; que el marco autonómico es central para su adecuado funcionamiento; que la coordinación a nivel estatal es una exigencia de todo sistema integrado, y que la acción comarcal y la participación popular es garantía de una política social y económicamente bien encauzada.

Desde el punto de vista de la acción regional la Comarca debe delimitarse según estos principios:

a) Espacio heterogéneo de actividades económicas.

b) Uno y más centros de atracción de distinto rango. 

mite.

c) El Municipio como base de la unidad espacial que se deli-

d) Que las unidades resultantes sean viables, o sea, equilibradas desde los puntos de vista de población y superficie.

La comarcalización propuesta del País Valenciano es la siguiente:

PROVINCIA DE CASTELLON

ELS PORTS DE MORELLA

LA TINENÇA DE BENIFASSAR-PLA

DE VINAROS

EL MAESTRAT

El Baix Maestrat-Pla del Arc

L'Alt Maestrat

L'ALCALATEN-PANYAGOLOSA

LA PLANA-MIJARES

La Plana de Castelló

Mijares

EL ALTO PALANCIA

PROVINCIA DE VALENCIA

LA SERRANIA-ADEMUZ

El Rincón de Ademuz

La Serranía del Turia

EL CAMP DEL TURIA

EL CAMP DE MORVEDRE

EL ALTIPLANO DE REQUENAUTIEL

LA HOYA DE BUNOL-CHIVA

L'HORTA
EL VALLE DE AYORA

LA CANAL DE NAVARRESENGUERA

LA RIBERA

La Ribera Alta

La Ribera Baja

LA COSTERA

LA VALL D'ALBAIDA

LA SAFOR

PROVINCIA DE ALICANTE

LES VALISS DEL VINALOPO

L'Alta Vinalopó

El Vinalopó Mitjá

L'ALCOIA

Els Valls d'Alcoi

La Foia de Castalla

LA MARINA ALTA

LA MARINA BAIXA

EL CAMP D'ALACANT-ELX

L'Alacantí

El Camp d'Elx

LA VEGA DEL SEGURA

\section{LA DIVISION COMARCAL DE RIBERA Y CABRER}

Tradicionalmente, al definir las unidades espaciales se han seguido tres tipos de criterios: a) Homogeneidad. b) Nodalidad. c) Programación práctica o administrativa.

Estadísticamente y desde las perspectivas homogénea y nodal pueden utilizarse las siguientes técnicas: a) Para delimitar Comarcas homogéneas se utilizan, por una parte, técnicas univariantes (una sola variable) y, por otra, técnicas multivariantes (varias variables simultáneas), entre las que cabe destacar el «Análisis de los componentes principales», el «Análisis de Cluster» y el «Análisis 
discriminante». b) Para delimitar Comarcas nodales, se utilizan las "Cadenas de Markov» y técnicas derivadas de la teoría de juegos.

El principio de homogeneidad permite que las unidades territoriales más pequeñas se agrupen de acuerdo con las similitudes que presentan respecto a una o varias variables-lengua, cultura, riqueza, actividad sectorial, infraestructura-.

Con arreglo al principio de nodalidad se parte del hecho de que sobre un territorio los factores económicos y humanos se sitúan alrededor de núcleos urbanos llamados «nodos», que dominan las áreas próximas y entre las que existen relaciones de interdependencia. Estos nodos pueden ser funcionales-mercado de trabajo, centros comerciales-o jerárquicos-administrativos, de comunicaciones-.

Mediante la programación práctica se trata de determinar las áreas administrativas y políticas con la finalidad de proporcionar las unidades territoriales que se utilizarán como instrumento político.

Con arreglo a estas técnicas, María José RiBERA OrTún y Bernardí CABrer Borrás han elaborado una comarcalización del País Valenciano, basada en el criterio económico, aunque sin limitarse exclusivamente a él.

En una primera fase reunieron una gran masa de datos referentes a cada uno de los 545 Municipios valencianos, mediante 22 indicadores económicos, utilizando la técnica del análisis multivariante y con auxilio de un ordenador.

La primera selección de variables, más amplia, se concretó finalmente en las siguientes:

Demográficas: Densidad.

Sector primario: Porcentajes de superficie dedicada a cultivos de regadío y de secano.

Sector secundario: Establecimientos industriales, licencias industriales, potencia industrial instalada y obreros por habitante.

Sector terciario: Cuota de mercado.

Consumo energético: Consumo de energía eléctrica industrial, residencial y de servicios.

Transporte y comunicaciones: Camiones y teléfonos por habitante.

Economía general: Bancos, Cajas de Ahorro, instrumentos notariales, presupuestos municipales, recaudación impuesto espectáculos.

Se delimitaron agrupaciones municipales basándose fundamen- 
talmente en razones geográficas, teniendo además en cuenta el área de influencia de los principales Municipios, que se han convertido a lo largo del tiempo en verdaderos centros neurálgicos de determinadas áreas.

Con esta información fundamental y mediante procedimientos matemáticos se obtuvo una comarcalización del País Valenciano, mediante el análisis de componentes principales, comprendiendo cada Comarca las agrupaciones siguientes:

PROVINCIA DE CASTELLON

I. Capital: Segorbe Segorbe Jérica-Viver

II. Capital: L'Alcora L'Alcora Atzeneta

III. Capital: Sant Mateu Albocáser Morella Sant Mateu

IV. Capital: Onda Onda

V. Capital: Vinarós Vinarós

VI. Capital: Vilafamés Vilafamés Coves de Vinromá Alcalá de Xivert

VII. Capital: Castelló Castello Borriana-Vila-Real Benicássim

VIII. Capital: Nules Nules

PROVINCIA DE VALENCIA

I. Capital: Ayora:Enguera Millares

Ayora Enguera
II. Capital: Buñol Chiva-Buñol

III. Capital: Requena Requena

IV. Capital: Albaida Ontinyent

Albaida

Benigánim

V. Capital: Xátiva

Xátiva

Moixent

VI. Capital: Gandía Gandía

VII. Capital: Alzira La Vall de Tavernes Sollana

Alzira

Alberic

Carlet

Llombai

VIII. Capital: Sueca Sueca

IX. Capital: Lliria Lliria

X. Capital: Valencia Bétera Valencia

XI. Capital: Villar del Arzobispo Chelva Villar del Arzobispo

XII. Capital: Sagunt Estivella Sagunt 
XIII. Capital: Ademuz Ademuz

PROVINCIA DE ALICANTE

I. Capital: Vila Joiosa

Benissa

Calp

Altea

Benidorm

Vila Joiosa

II. Capital: Alacant

Xixona

Alacant

III. Capital: Alcoi Alcoi
IV. Capital: Denia

Denia

Pego

Callosa d'En Sarriá

V. Capital: Elda-Villena-Ibi

Ibi

Elda

Villena

VI. Capital: Novelda

Novelda

VII. Capital: Elx

Elx

VIII. Capital: Orihuela

Orihuela

Almoradí

Torrevieja

De esta forma, el País Valenciano queda estructurado en 29 Comarcas.

Aplicando a las Agrupaciones iniciales la técnica del Análisis Cluster se configuran 30 Comarcas. En la Provincia de Castellón son igualmente ocho las Comarcas, aunque con algunas diferencias respecto a las derivadas del análisis de los principales componentes. Sólo se mantienen invariables las Comarcas de Segorbe y Vinaroz. La segunda tiene por capital a L'Alcora y de ellas forman parte las agrupaciones de L'Alcora y Onda. La tercera, con Sant Mateu como capital, comprende las Agrupaciones de Sant Mateu, Coves de Vinromá y Alcalá de Xivert. La cuarta Comarca es Morella, con su propia Agrupación. La quinta es Albocásser, con su propia Agrupación. La séptima, Vilafamés, con las Agrupaciones de Atxeneta, Vilafamés y Benicássim. Y la octava Castelló, con las Agrupaciones de Castelló, Vila-Real-Borriana y Nules.

En la Provincia de Valencia, las Comarcas, según el Análisis de Cluster, siguen siendo 13 , con idéntico contenido, en cuanto a las Agrupaciones de cada Comarca que las configuradas con el análisis de los principales componentes, salvo que la Comarca de Valencia tiene su propia Agrupación y la Agrupación de Bétera se incorpora a la Comarca de Sagunt.

En la Provincia de Alicante, el número de Comarcas pasa a ser de nueve. La novena Comarca se constituye con las Agrupaciones 
160

REVISTA DE ESTUdIOS DE LA VIDA LOCAL

de Xixona e Ibi, sacadas de las Comarcas segunda y quinta anteriores.

Como se ha observado, salvo en la Provincia de Castellón, los resultados fueron prácticamente similares en los procedimientos de análisis aplicados.

Los autores, finalmente, como conclusión de su estudio, proponen, en definitiva, las siguientes Comarcas:

\begin{tabular}{|c|c|}
\hline PROVINCIA DE CASTELLON & Buñol \\
\hline Morella & Ayora-Enguera \\
\hline Albocásser & $\begin{array}{l}\text { Alzira } \\
\text { Xátiva }\end{array}$ \\
\hline Llucena & Albaida \\
\hline $\begin{array}{l}\text { Segorbe } \\
\text { Sant Mateu-Vinaros }\end{array}$ & Gandía \\
\hline Castello & PROVINCIA DE ALICANTE \\
\hline PROVINCIA DE VALENCIA & $\begin{array}{l}\text { Denia } \\
\text { Vila Joiosa }\end{array}$ \\
\hline Ademuz & Alcoi \\
\hline Villar del Arzobispo & Villena-Ibi-Elda \\
\hline Lliria & Alacant \\
\hline Sagunt & Novelda \\
\hline Valencia & Elx \\
\hline Requena & Orihuela \\
\hline
\end{tabular}

En total, 26 Comarcas, cuya configuración se asemeja mucho a las Comarcas tradicionales. 


\section{REVISTA}

DE IUDIOS
DST LA
DE LA
VIDA LOCAL

\section{JURISPRUDENCIA}


REVL-1981, núm. 209. BALLESTER ROS, IGNACIO. LA DIVISION TERRITORIAL EN COMARCAS...

REVL-1981, núm. 209. BALLESTER ROS, IGNACIO. LA DIVISION TERRITORIAL EN COMARCAS... 